\title{
The benefits of executive control training and the implications for language processing
}

\author{
Erika K. Hussey ${ }^{1,2}$ and Jared M. Novick ${ }^{1,2}$ \\ ${ }_{1}^{1}$ Program in Neuroscience and Cognitive Science, Department of Psychology, University of Maryland, College Park, MD, USA \\ ${ }^{2}$ Center for Advanced Study of Language, University of Maryland, College Park, MD, USA
}

Edited by:

Andriy Myachykov, University of

Glasgow, UK

Reviewed by:

Silvia P. Gennari, University of York, UK Marina Bedny, Massachusetts

Institute of Technology, USA

*Correspondence:

Erika K. Hussey, Program in Neuroscience and Cognitive Science, Department of Psychology, University of Maryland, 1147 Biology-Psychology Building, College Park, MD 20916, USA.

e-mail: ehussey@umd.edu
Recent psycholinguistics research suggests that the executive function (EF) skill known as conflict resolution - the ability to adjust behavior in the service of resolving among incompatible representations - is important for several language processing tasks such as lexical and syntactic ambiguity resolution, verbal fluency, and common-ground assessment. Here, we discuss work showing that various EF skills can be enhanced through consistent practice with working-memory tasks that tap these EFs, and, moreover, that improvements on the training tasks transfer across domains to novel tasks that may rely on shared underlying EFs. These findings have implications for language processing and could launch new research exploring if EF training, within a "process-specific" framework, could be used as a remediation tool for improving general language use. Indeed, work in our lab demonstrates that EF training that increases conflict-resolution processes has selective benefits on an untrained sentence-processing task requiring syntactic ambiguity resolution, which relies on shared conflict-resolution functions. Given claims that conflict-resolution abilities contribute to a range of linguistic skills, EF training targeting this process could theoretically yield wider performance gains beyond garden-path recovery. We offer some hypotheses on the potential benefits of EF training as a component of interventions to mitigate general difficulties in language processing. However, there are caveats to consider as well, which we also address.

Keywords: cognitive training, executive function, conflict resolution, process-specificity, language processing, ambiguity resolution

\section{INTRODUCTION}

Cognitive control, also called executive function (EF), refers to a cluster of mental processes that permit the flexible adjustment of thoughts and actions across domains, allowing individuals to adapt to new rules and guide the selection of task-relevant over task-irrelevant information in an environment that varies continuously (Miller and Cohen, 2001). As we navigate our surroundings, we can frequently rely on a set of highly regularized functions that render certain tasks like driving a car or skimming a magazine article relatively automatic. Sometimes, however, new instructions or conflicting information compels us to override these reflexive actions and instead consider what might otherwise be a disfavored (or atypical) response. For instance, a resident of Chicago may be in the habit of making a legal right turn on red when driving at home, but this routine behavior could result in a costly ticket when she visits New York City, where turning on red is strictly prohibited! Likewise, imagine reading the following sentence upon skimming a magazine: at the restaurant, the interns discussed the bill before suggesting edits to the senator. One might initially interpret the word "bill" to mean the list of charges incurred for the meal, rather than its intended (though less common) interpretation, namely a draft piece of legislation. On the surface, both examples are quite different, but conceivably induce a similar experience: the detection of an incompatibility and the ensuing need to rein-in a highly familiar, yet currently inappropriate cognitive reaction (e.g., refrain from turning; revise the more frequent meaning, but current misanalysis, of "bill"). Such "conflict resolution" functions are an essential part of cognitive control (Botvinick et al., 2001) and help adapt information-processing strategies so individuals can regulate behavior in view of ever-changing goals, new contexts, or situation-specific demands.

As many researchers have argued, EFs encompass a collection of cognitive processes that help guide goal-directed behavior; that is, cognitive control is not a unitary construct but comprises separable components (Norman and Shallice, 1986; Botvinick et al., 2001; Miller and Cohen, 2001). In addition to the conflict-resolution processes outlined above, other EFs include task-switching, updating, and information monitoring, each of which can operate over visual, spatial, or verbal domains (Smith and Jonides, 1999; Miyake et al., 2000; Friedman and Miyake, 2004) and thus may be recruited across a variety of tasks including selective attention, decisionmaking, working memory (WM), error monitoring, and language processing (Botvinick et al., 2001; Thompson-Schill et al., 2005; Badre and Wagner, 2007; inter alia). With regard to conflictresolution functions in particular, converging data from neuropsychological patients and brain-imaging studies of healthy adults suggest that, across a range of WM, attention, and language tasks, posterior regions of left ventrolateral prefrontal cortex (VLPFC) commonly support the ability to resolve among competing sources of evidence, regardless of domain (Thompson-Schill et al., 2005). 
In this paper, we discuss how a burgeoning literature demonstrates that EFs can be trained through ample practice - that such abilities are seemingly not fixed, but malleable - and that performance increases throughout the course of training generalize to novel tasks that were not part of the training protocol. Some examples of transfer include benefits on unpracticed tasks tapping fluid intelligence (Jaeggi et al., 2008), working-memory updating (Dahlin et al., 2008; Li et al., 2008), and task-switching (Karbach and Kray, 2009) - that is to say, transfer benefits have been observed across a range of EF.

We are especially interested in the implications that these training-transfer findings have for language processing under conditions of conflict, given that domain-general conflict-resolution and cognitive-control functions have been associated with assorted linguistic abilities including the resolution of lexical (Bilenko et al., 2009; Copland et al., 2009; Khanna and Boland, 2010) and syntactic ambiguities (Novick et al., 2005), verbal fluency (Robinson et al., 1998; Kan and Thompson-Schill, 2004; Novick et al., 2009; Schnur et al., 2009), and perspective-taking during natural dialog (Brown-Schmidt, 2009; Nilsen and Graham, 2009; for reviews, see Novick et al., 2005; Novick et al., 2010). Thus, in the hypothesis section, which details the potential implications of EF training and transfer effects on language use, we consider a theory based on evidence that left VLPFC-supported conflict resolution is the kind of cognitive-control function of principal relevance to these particular linguistic tasks (see e.g., Novick et al., 2005). We couch our hypotheses within a process-specific account (see Dahlin et al., 2008; Shipstead et al., 2010, 2012), which in the training literature posits that post-intervention, performance increases on novel tasks largely depends on the extent of overlap between the training and transfer measures, both in terms of the shared cognitive processes and underlying neural systems needed to complete them. That is, if a certain component of EF (e.g., conflict resolution) is targeted and improved through training, then transfer measures relying on common processes should be influenced accordingly, irrespective of domain. In view of this, we will focus our discussion on a few language comprehension and production tasks that fit within the VLPFC-mediated process-specific function typically referred to as "conflict resolution." However, in the discussion, we acknowledge other brain systems involved in a wider array of EFs, and consider briefly the implications for training and the effects on language.

As sketched in the driving and reading examples earlier, when we talk about conflict (or interference), we are referring to conditions that contain the presence of mismatched or incongruent sources of evidence. Specifically, "conflict" designates cases in which current situation-specific demands generate an incompatibility between how an input stimulus should be characterized (dubbed representational conflict), given how the input is normally considered. Such conflict is often called "prepotent conflict," because individuals must override their dominant (prepotent) biases in support of atypical alternatives (Botvinick et al., 2001). For instance, the Stroop task is a canonical representational conflict task involving the need to countermand a prepotent bias that is generated by a lexical representation (which gives rise to an automatic reading response), in favor of a perceptual (color) representation. A comparable type of representational conflict occurs in the form of "underdetermined conflict," in which multiple candidate representations are equally reasonable and thus compete for selection (Botvinick et al., 2001). Importantly, brain-imaging findings suggest separable neuroanatomical involvement for representational conflict versus response conflict (or response selection; see Milham et al., 2001; Nelson et al., 2003). Our major focus here is on the implications of conflict-resolution training at the representational level on particular language-performance measures such as lexical and syntactic ambiguity resolution (in comprehension) and verbal fluency (in production). Both prepotent and underdetermined representational conflicts recruit posterior regions of VLPFC (Brodmann areas 44 and 45) across language and memory domains, meeting the requirements for a test of process-specificity (see Novick et al., 2010; see also Milham et al., 2001 and Nelson et al., 2003, which demonstrate VLPFC recruitment for representational conflict resolution but anterior cingulate recruitment for response-level conflict resolution).

Generally, we believe that - considering the mounting evidence showing the effectiveness of various types of EF training in different populations (Klingberg et al., 2005; Westerberg et al., 2007; Jaeggi et al., 2011) - there is room to establish new research investigating if EF training protocols that focus on selective subprocesses (i.e., representational conflict resolution) could be used successfully as an intervention technique to mitigate problems in general language use that arise under high-EF (i.e., high-conflict) demands.

Indeed, there is tantalizing evidence supporting processspecific transfer to conflict-related language measures, drawn not from a long-term training paradigm per se, but rather from another type of intervention designed to fatigue selective cognitive processes common to WM and language processing tasks. These so-called "resource depletion models" offer an interesting framework to understand negative transfer to tasks relying on temporarily exhausted EFs shared across ostensibly different domains (Van der Linden et al., 2003; Persson et al., 2007). That is, rather than boosting general-purpose EFs through long-term practice, as is the case with training studies, resource depletion paradigms rely on short-term "overuse" of a particular cognitive process. For example, after performing a complex task that places high demands on EF capacities, these resources are rendered temporarily unavailable for continued use; therefore, performance decreases on transfer measures that rely on the common "worn out" EF (Van der Linden et al., 2003; Persson et al., 2007; see also Snyder et al., 2010 for similar findings among anxious individuals).

In one study (Persson et al., 2007), conflict-resolution abilities were fatigued through an intensive session of an item-recognition task with high conflict-resolution demands. In this task, participants indicated whether a probe item (e.g., $C$ ) appeared in an immediately prior memory set (e.g., $r, f, c, l$; see Monsell, 1978). Frequently, subjects could respond correctly due to familiarity alone: familiar probes required a "yes" response and unfamiliar ones a "no" response. However, relying on familiarity on some "no" trials was prone to error, because they contained a probe (e.g., $G$ ) that was not among the current memory set $(j, p, v, m)$ but was among the items in the prior trial $(g, k, v, p)$. Thus, these trials required subjects to override a prepotent familiarity bias (and "yes" response) and instead re-characterize the probe stimulus as 
"familiar-but-irrelevant," and respond "no." Such "recent-no" trial types, when compared to "non-recent-no" trials (when the probe did not appear in either the current or preceding sets) routinely recruit left posterior VLPFC (Jonides and Nee, 2006). Important for the current discussion, after subjects completed this task and "fatigued" the conflict-resolution process, they subsequently demonstrated selective performance decline on VLPFC-mediated, high-conflict conditions on a verbal fluency task, in which they had to generate an associated verb to a given noun (e.g., scissors $\rightarrow$ cut; high-conflict items had many possible associated verbs, like ball $\rightarrow$ kick, throw, catch, bounce, and thus contained underdetermined response conflict; see Thompson-Schill et al., 1998). This pattern of negative transfer was not observed for (1) subjects who received exposure to only low-conflict trials during their intensive practice session (i.e., no recent-no trials were present); or (2) individuals who practiced a different task before the verb generation task, namely a stop-signal task that recruits mainly right-hemisphere networks and a different subcomponent of EF (response inhibition; see also Friedman and Miyake, 2004). Together, this suggests that the process-specificity observed across intervention and transfer tasks operates on a short time scale, such that as conflict resolution is temporarily depleted, other tasks relying on shared cognitive and neural resources are affected accordingly.

Although these effects are transient, the selective transfer findings are nonetheless critical: they demonstrate that conflict resolution abilities are at least temporarily malleable, and this malleability can subsequently affect language processing under similar conditions of high conflict. Consequently, we ask: considering evidence for process-specific transfer, on a short time scale, across memory and language tasks that commonly rely on VLPFCmediated conflict-resolution functions, might one observe longerterm effects on language measures as well, when conflict resolution is boosted via extensive practice? That is, can we observe positive transfer - namely, performance increases - when individuals consistently train conflict-resolution functions over time? We hypothesize that the answer should be yes, given the evidence that other EFs (e.g., task-switching, etc.) are both trainable and transferrable. Indeed, work from our lab demonstrates reliable transfer to syntactic ambiguity resolution in healthy adults, where individuals who have undergone extensive conflict-resolution training fare significantly better at revising early misinterpretations than their untrained counterparts (Hussey et al., 2010; Novick et al., submitted for publication). Additionally, on the basis of the theory that posterior regions of VLPFC support conflict resolution across domains, such displays of transfer, we hypothesize, might clearly extend beyond just "garden-path" recovery, given the putative role of conflict-resolution in several other measures of language processing.

Although we outline below some potential benefits of conflictresolution training on language use, we also discuss some caveats that should be considered, including individual differences in training success (not everyone responds to training or achieves similarly high levels, cf. Chein and Morrison, 2010; Jaeggi et al., 2011), limitations that may be involved in training special populations, and the need for explicit linking hypotheses between training and any expected transfer: namely, there must be a theory that bridges the hypothesized underlying cognitive processes from one task to another (i.e., from an intervention task to a transfer task). Transfer from training to untrained assessment tasks cannot be expected, or explained, without a well-formulated process-specific theory (Shipstead et al., 2010, 2012). To this end, we also speculate that the magnitude of transfer effects is contingent upon the degree to which a targeted EF contributes to and shares critical features with an outcome measure. This is particularly important if, as some researchers suggest, EF is not a unitary construct but is comprised of separable, multi-component processes such as conflict resolution, updating, and task-switching (Miyake et al., 2000; Persson et al., 2007; Dahlin et al., 2008).

As outlined in this hypothesis and theory piece, we integrate the extant training and psycholinguistic literatures to develop testable hypotheses from an emerging picture within the EF training research. The following section begins with a brief review of cognitive training studies demonstrating transfer to novel tasks that are ostensibly different from those practiced during the training regimens, but share specific processing demands. We then turn to research on the role of conflict resolution in language use, sketching some hypotheses and implications the training findings have for new work aimed at improving language processing under high-EF - particularly high-conflict-resolution demands. That is, if conflict-resolution is malleable (which seems to be the case given the resource depletion work outlined above), we hypothesize that training such processes should also show transfer to untrained measures of conflict resolution within the linguistic domain, patterning with other training-transfer findings. The theory bolstering this claim comes from work (drawn from patients, children, and brain-imaging studies of adults) indicating that conflict-resolution and cognitive-control measures play an important role in language tasks that we outline below.

\section{EXECUTIVE FUNCTION TRAINING AND ITS TRANSFER ACROSS COGNITIVE DOMAINS: A BRIEF REVIEW}

A recent flurry of research is devoted to testing if general-purpose cognitive abilities can be enhanced through consistent practice with WM tasks that recruit brain regions within the corticostriatal network key to executive functioning. Although interventions geared toward improving psychological faculties, specifically intelligence, were pioneered decades ago (see Feuerstein, 1980), Klingberg and colleagues have recently reinstated the notion by training domain-general cognitive abilities as a means to remediate populations with diminished WM resources including stroke patients (Westerberg et al., 2007), children with attention deficit hyperactivity disorder (Klingberg et al., 2005), and older adults (Brehmer et al., 2011). Ever since, cognitive training programs have undergone significant study, particularly in healthy adults, to examine whether normally functioning individuals' EF abilities can be improved, and what generalized outcomes consistent training might have on everyday performance on non-trained tasks. To this end, researchers have been investigating questions related to dosage-dependence (does more practice yield more transfer?; Jaeggi et al., 2008), the extent to which training transfers to untrained but related measures (Li et al., 2008; Karbach and Kray, 2009; Chein and Morrison, 2010; Morrison and Chein, 2011), if training tasks must adapt to individuals' performance 
to be effective (Klingberg et al., 2005; Brehmer et al., 2011), and individual differences in training success (Jaeggi et al., 2011).

Here, we focus on the extent to which training generalizes to novel tasks. The typical training study is designed as a pre/post longitudinal experiment in which subjects are assessed on some cognitive capacity immediately before and again after an extensive intervention. In some cases, the intervention comprises practice with a single training task (Dahlin et al., 2008; Jaeggi et al., 2008, 2011; Li et al., 2008), whereas in others, a battery of training tasks is administered (Klingberg et al., 2002, 2005; Karbach and Kray, 2009). Regardless, the training tasks are different from those completed at the pre/post assessment sessions, with the intervention component typically lasting for several hours distributed over a few weeks. Upon conclusion of the regimen, trainees return to the lab and complete follow-up assessments, namely complementary versions of the tasks that were done just prior to training, to evaluate whether performance on assessments has reliably improved, thereby providing evidence for "transfer."

Transfer has been documented for untrained tasks that share obvious features with well-practiced training tasks, an effect sometimes referred to as "near-transfer." For instance, performance increases on WM training tasks generalize to structurally similar (but new) WM assessments (Li et al., 2008; Karbach and Kray, 2009; see below). However, "far transfer" can also be observed, namely to assessments that appear, on the surface, to be wildly different from the training tasks completed throughout the intervention regimen (Kloo and Perner, 2003; Dahlin et al., 2008; Jaeggi et al., 2008, 2011). This latter form of transfer is possible provided that training and assessment tasks share certain essential underlying EFs (as well as overlapping neural resources; see Jonides, 2004; Shipstead et al., 2010, 2012).

\section{NEAR-TRANSFER OF TRAINING}

Near-transfer effects emerge when the nature of the processed information - including stimulus type, task structure, and response type - is similar across training and assessment tasks (but see Morrison and Chein, 2011 for an alternative definition of near-transfer). For instance, in one report (Li et al., 2008), trainees practiced a spatial 2-back task, during which they had to monitor the locations of sequentially presented squares on a $3 \times 3$ grid and respond whenever the current location matched the location seen two trials earlier. Compared to a no-contact control group, trained participants demonstrated post-intervention improvements on a spatial 3-back task, providing evidence for near-transfer to a more difficult, but otherwise identical task. Another type of neartransfer occurs when the type of information (i.e., the stimuli) being processed is changed across training and transfer tasks, while the response-level requirements remain constant, resulting in a structural continuity between both tasks. For example, in the same study by Li et al. (2008), trainees also improved on numeric 2- and 3-back tasks, where instead of remembering locations on a grid, subjects indicated when a serially presented number $(0-$ 9) matched the identity of a number presented two (or three) trials previously. The authors argued that transfer to a numeric $n$-back task provided support for a task-specific response strategy shared across stimulus modalities: Although the spatial 3-back and numeric $n$-back tasks differ from the spatial 2-back training task, all require the same basic strategy, namely, information must be monitored and updated in a predictable fashion.

In addition to the above findings, Karbach and Kray (2009) observed that increases in task-switching abilities - an EF based on mental shifting across different goals or rules - as a consequence of training generalizes to performance on novel tasks with similar switching demands. Specifically, their training regimen involved making two-alternative forced-choice judgments about pictures (trees/flowers), based on two separate characteristics (e.g., identity vs. color), such that the relevant characteristic (or rule) changed predictably across trials. Stimulus types (fish/birds, trees/flowers, sports/music, planes/cars) and response categories (identity, number, color, and rotation) varied across sessions within the training regimen. An assessment of near-transfer involved responding to a novel set of stimuli (fruits/vegetables) using number and identity as response categories; compared to a non-switching active-control group, the task-switching trainees showed greater posttest improvement in switching costs, i.e., the difference in response time on switch (color followed by identity judgment) vs. non-switch trials.

These examples highlight two sources of near-transfer: training and outcome measures tap the same underlying EFs (e.g., monitoring and updating), and both tasks provoke similar processing demands through a shared task structure (task-specific aspects). Consequently, it is difficult to disentangle the source of near-transfer effects, as two possibilities may account for any observed pre/post changes: (1) the trained EF shared by both tasks may have been improved, or (2) a task-specific strategy may have been developed. Indeed, in cases of near-transfer, the training and transfer tasks need not tap the same underlying EFs, since transfer could occur simply with improvements at task-specific aspects of the paradigm. Near-transfer effects might be unsurprising: practicing an $n$-back task improves $n$-back performance, and therefore transfers to other $n$-back tasks (perhaps regardless of domain); likewise, practicing a categorization task-switching task generalizes to a similar task with novel categories. But, the extent to which these near-transfer effects are driven by the shared EFs across training and assessment tasks, the surface-level features (stimulus or response characteristics) that are isomorphic between both sets of tasks, or through a combination of both factors is unknown.

\section{FAR-TRANSFER OF TRAINING}

Training studies designed to show far-transfer effects help to elucidate the role of shared EFs; by design, the surface-level properties - stimuli or required responses - of the training and assessment tasks are quite different. Consequently, contrary to near-transfer findings, far-transfer effects are assumed not to rely heavily on the structural (task-specific) similarities across training and assessment tasks, and instead result mostly from improvements on underlying EFs important to both the training and assessment measures (Shipstead et al., 2010). In other words, the goal of fartransfer training is rooted in improvement of specific processes engaged during tasks with dissimilar structures, often spanning domains (again, sometimes referred to as process-specific training).

For instance, in one set of studies, subjects practiced a dual $n$-back memory task involving simultaneous updating of shape locations and the identity of heard letters, such that a target was 
defined as an item repeating $n$-trials previously in either modality (Jaeggi et al., 2008). Trainees showed subsequent improvements on Raven's Advanced Progressive Matrices, a transfer task that requires participants to select a textured shape from a set of possible response items, which fits a sequence of other textured shapes to complete a particular pattern with one absent piece (Jaeggi et al., 2008, 2011). The response and surface-level properties of $n$-back and Raven's are distinct, as one task involves monitoring a continuous stream of letters or block locations for familiar instances, and the other requires reasoning to identify the missing element that completes a $4 \times 4$ matrix containing orderly patterns across rows and columns; thus, to observe transfer, there must be an underlying process common to both tasks that is enhanced through intensive $n$-back training. The authors reasoned that this shared process centered around a common need to employ attentional control, such that their training procedure - which forced trainees to practice constant shifting of attention to new stimulifacilitated this ability, thereby enabling transfer to Raven's, which similarly involves updating and selection among multiple representations (via the control of attention). Importantly, because the training and transfer measures were characteristically so different, the authors argued that task-specific elements could not explain the observed generalization, effectively ruling out near-transfer as an explanation for their findings. Rather, training boosted a part of the EF system - here, multiple-task management and attentional control processes - important for a range of cognitive tasks, including Raven's performance. Indeed, separate work demonstrates that $n$-back and Raven's activate a similar network of neural regions, providing additional support for resources common to both tasks (Burgess et al., 2011).

Additional evidence of process-specific training comes from demonstrations of selective far-transfer from an updating task (letter running-span) to a structurally different assessment measure (number $n$-back) that requires a similar updating EF; critically though, such transfer was not demonstrated on the Stroop task, which relies on a separable EF - conflict resolution (Dahlin et al., 2008). During the letter running-span task, participants must recall the last four items of a study list that terminates unexpectedly, forcing them to continuously update the correct response set from a fleeting memory store; similarly, their version of $n$ back required subjects to monitor and refresh representations as new information is processed and deemed relevant. Running-span and a standard number $n$-back task recruit similar striatal regions, corroborating their underlying reliance on a common EF. Contrastingly, tasks requiring conflict resolution, like Stroop, require subjects to re-characterize an automatized response (reading) in order to promote atypical, but task-relevant information (color name); such tasks rely on a separable neural profile (compared to that required for updating tasks) including a network of frontal and parietal regions. Dahlin et al. (2008) demonstrated that training on running-span confers benefits to assessment measures that share updating demands and corresponding neurological profiles ( $n$-back), while those with little or no such overlap (Stroop) show negligible improvement. In sum, the amount of far-transfer to untrained tasks following intervention depends on the degree of overlap among cognitive and neural resources shared by the training and the transfer tasks.
Given these training and far-transfer effects for a range of EFs (e.g., attention control, memory updating), one might also hypothesize that transfer from general-purpose EF training to certain tasks of language processing might occur as well. That is, the language tasks are not trained per se, but tap particular cognitive functions (conflict resolution) that may be trainable through an extensive regimen targeting common processes (or neural resources). As hypothesized below, the result could be an alleviation of language processing difficulty under conditions that place heavy demands on the EF system in healthy, and perhaps even in special populations. We focus on a select few of these language conditions in the following section, concentrating specifically on a functional-anatomical association between conflict-resolution processes of EF, and regions within left VLPFC that support them (for an extensive review, see Novick et al., 2010). We sketch how this association is important for production and comprehension abilities in healthy adults, young children, and patients with circumscribed VLPFC damage.

\section{THE ROLE OF EXECUTIVE FUNCTION IN LANGUAGE USE: HYPOTHESES AND IMPLICATIONS FOR TRAINING}

One priority in psycholinguistics has been to study how nonlinguistic cognitive abilities contribute to language production and comprehension. EF abilities have emerged as a candidate characteristic, defining in part those individuals who can better coordinate rapidly among multiple sources of linguistic (syntactic, semantic) and extra-linguistic (pragmatic, contextual) evidence across a range of communicative tasks. Given the breadth of work on various EFs for language, we focus only on the role of conflict-resolution training for a handful of language tasks. As sketched in the introduction, conflict resolution refers to the recharacterization of information in the face of competing sources of evidence. Regarding language processing, good conflict-resolution skills enable readers and listeners to avoid comprehension errors in the face of ambiguity (e.g., by consulting top-down evidence to override misinterpretations), produce the right word among competing options, and take an interlocutor's perspective when assessing common-ground information during natural, unscripted dia$\log$ (see Novick et al., 2005, 2010). Indeed, patients with circumscribed damage to left posterior VLPFC consistently underperform on high-conflict conditions on non-linguistic tasks such as Stroop and the "recent-no" task described above (Hamilton and Martin, 2005). Moreover, this general conflict-resolution disorder in patients has been tied to their concomitant deficits on language tasks that generate similar conflict-resolution demands, for example, when dominant meanings of lexical ambiguities must be countermanded (Bedny et al., 2007), when initial interpretations of syntactic ambiguities must be reprocessed (Novick et al., 2005, 2009), or when object names must be selected among categorical competitors (Schnur et al., 2009). As such, by training generalpurpose conflict-resolution abilities - supported by regions within VLPFC - in healthy adults, we hypothesize that there should be systematic improvements in high-conflict conditions on language tasks requiring shared demands for conflict resolution. Below, we provide examples of when conflict-resolution abilities appear to 
interact with particular language processing skills and outline the implications these associations have for process-specific training.

\section{SYNTACTIC AMBIGUITY RESOLUTION \\ Theory}

Readers and listeners process sentences in real-time, committing to an interpretation incrementally as words and phrases are encountered moment-by-moment (Altmann and Kamide, 1999; Tanenhaus, 2007). One consequence of incremental processing is temporary ambiguity: the first analysis individuals assign sometimes turns out wrong. Cognitive control has been tied to individuals' ability to adjust interpretations when late-arriving evidence signals that their initial analysis was incorrect (Novick et al., 2005). Such cases of conflict (the so-called "garden-path effect") elicit temporary processing difficulty in reading (Frazier and Rayner, 1982; Staub and Rayner, 2007; inter alia) and confusion during spoken comprehension (Tanenhaus et al., 1995). Individuals must then engage in a process that permits them to revise and capture the intended interpretation.

Evidence for the role of conflict-resolution in this recovery process comes from populations with underdeveloped or impaired cognitive control such as young children (whose PFC development is protracted; see Huttenlocher and Dabholkar, 1997) and patients with focal damage to left posterior VLPFC. Both populations fail to initiate cognitive-control functions across assorted non-syntactic measures (e.g., Stroop, the recent-no, and other analogous tasks; e.g., Hamilton and Martin, 2005; Khanna and Boland, 2010), and both groups similarly fail to revise sentence interpretations following early misanalysis (Trueswell et al., 1999; Weighall, 2008; Novick et al., 2009; see also Christianson et al., 2006 for similar patterns in older adults). The linking assumption is that the discovery of a misinterpretation deploys conflict-resolution to resolve the incompatibility between representations of sentence meaning: the one initially assigned and the one in need of recovery, similar to the controlled processes required to resolve conflict during incongruent Stroop trials, or interference from familiar but currently irrelevant items in the "recent-no" task (Hamilton and Martin, 2005; Novick et al., 2005, 2010). Interestingly, healthy adults undergoing functional neuroimaging demonstrate co-localized neural activity within left posterior VLPFC when performing both syntactic and non-syntactic tasks requiring conflict resolution, corroborating the necessary involvement of shared, domain-general processes presumed from special populations (January et al., 2009; Ye and Zhou, 2009).

\section{Hypothesis}

This convergence of findings suggests an opportunity to alleviate the processing difficulty associated with temporary ambiguities that arise during sentence processing by targeting the EFs (through training) that appear to be domain-general, i.e., common across certain syntactic and non-syntactic tasks. We tested this hypothesis in a study in which healthy trainees completed pre/post reading assessments involving syntactically ambiguous sentences susceptible to misanalysis (Hussey et al., 2010; Novick et al., submitted for publication). We hypothesized that practicing a performance-adaptive non-linguistic task requiring conflictresolution processes - the $n$-back memory task with lures (see below) - would endow trainees with improved abilities essential to re-interpreting garden-path sentences. (Performance adaptation means that as subjects reached a certain criterion, task difficulty increased dynamically in terms of $n$ and the number of lures present.) Similar to the processing demands of the recent-no task, our training task required participants to re-characterize stimulus representations in real-time. Specifically, subjects completed a version of $n$-back during training that contained interference lures, or items that match in target-identity but appeared in non$n$-positions. For example, in the sequence $G-P-K-G$, the second $G$ would be a target in a 3-back condition because it matches the 3back stimulus. However, in the sequence $G-P-K-L-G$, the second $G$ would be a "lure" in a 3-back condition because it matches the stimulus presented four, not three, items back (Gray et al., 2003; Burgess et al., 2011). We argued (as have others) that the familiarity of lure items forces participants to engage conflict-resolution functions to override a familiarity bias and the tendency to respond "target" to familiar representations; instead, subjects must re-characterize familiar letters in non- $n$ locations as non-targets (thus lures are akin to "recent-no" trials in the item-recognition task). Importantly, neuroimaging work (Gray et al., 2003) demonstrates that lure trials activate VLPFC resources that are also recruited during high-conflict language processing tasks. This finding suggests that practicing an $n$-back task with lures may lead to improvements not just on that task, but also in resolving competing interpretations of syntactically ambiguous sentences.

To examine process-specific training-related changes in sentence processing, readers' eye movements were recorded; we were primarily interested in the effect of training on processing difficulty, particularly in sentence regions that introduced new evidence signaling an incompatibility with individuals' early interpretations (i.e., disambiguating regions that induce conflict). Readers also answered comprehension questions, the responses to which indexed a failure to ultimately override their original misanalysis (Christianson et al., 2006). We found three important patterns: (1) those trainees who responded most to n-back practice reflected in steady performance gains throughout the regimen demonstrated significantly improved comprehension accuracy at posttest for ambiguous (but not unambiguous) materials, whereas the untrained controls and non-responsive trainees did not; (2) responsive trainees' reading times were reliably faster at posttest, acutely in disambiguating regions of ambiguous sentences, but not in other regions, reflecting less processing difficulty post-training upon encountering conflicting evidence - the control group and non-responders demonstrated no test-retest change; and (3) trainees' performance improvement on $n$-back-with-lures - and no other training task administered as controls - predicted the increases they achieved in garden-path recovery.

The selectivity of these findings is of particular interest, because trainees exhibited improvements only on the language materials where conflict-resolution processes are hypothesized to trigger (unambiguous materials did not involve the need to employ control to revise interpretations, and no test-retest changes in accuracy or reading times were found in this condition). Further, these pre/post improvements were accounted for only by individual training gains on the $n$-back-with-lures task - i.e., a task requiring conflict resolution - and no other well-practiced 
WM task completed during intervention (participants also trained on tasks tapping visuo-spatial and verbal WM functions without conflict-resolution demands). Importantly, many researchers argue (D'Esposito and Postle, 1999; Kane and Engle, 2000) that there are some tasks of WM that tap non-mnemonic functions, such as the need to resolve conflicting representations, which is a general-purpose skill necessary for some (not all) WM tasks and some language tasks like syntactic ambiguity resolution (Novick et al., 2005).

Overall, the patterns are consistent with the idea that the ability to recover from misinterpretation can be enhanced by training domain-general EFs common to some tasks of language processing and some tasks of WM. These findings indicate that within the right framework, and having appropriate linking hypotheses, EF training may be a viable way to improve language use under certain conditions through tests of far-transfer. Open questions remain about the trainability of special populations - particularly if training VLPFC patients and young children with poor conflict-resolution skills will result in improved cognitive control, extending to an enhanced ability to recover from parsing misanalyses. But the opportunity to test such ideas is ripe. To our knowledge, this study is the first to investigate the impact of EF training on the processes that commonly contribute to language comprehension. As sketched below, conflict-resolution abilities are associated with various other specific language processing tasks, leaving room to explore the effects of training on language use more generally.

\section{LEXICAL AMBIGUITY RESOLUTION}

\section{Theory}

Research examining comprehension at the single-word level suggests a role for conflict resolution when the dominant meaning of an ambiguous word (e.g., bill, as the tab issued by a restaurant) must be overridden to retrieve its subordinate meaning (an outline of a prospective law; Bedny et al., 2007). Questions posed in this literature examine whether good conflict-resolution skills enable context-dependent meaning selection, and conversely, whether poor abilities impair it. Researchers have found that better conflict resolution is related to young children's contextual sensitivity: context can be used by kids to countermand dominant, but inappropriate meanings of an ambiguous word; however, the use of top-down information is largely dependent on the maturity of their EF abilities, as indexed by a separate task of conflict resolution and inhibitory control (Khanna and Boland, 2010). Correspondingly, neuropsychological patients with poor conflict resolution show inadequate lexical ambiguity resolution when the subordinate meaning is activated by local contextual information (Balota and Faust, 2001; Bedny et al., 2007), suggesting that such patients have difficulty suppressing context-inappropriate meanings of ambiguous words (Copland et al., 2009; Vuong and Martin, 2011). Finally, across several studies, regions within VLPFC - the same areas involved in lesion-deficit analyses of patients showing conflict-resolution impairments - are active in healthy adults during lexical-decision tasks necessitating resolution of meaning competition, suggesting that VLPFC-mediated EFs trigger to resolve increased competition associated with accessing the less frequent meaning of an ambiguous word (Bilenko et al., 2009).

\section{Hypothesis}

Considering the training results observed for syntactic ambiguity resolution - and therefore assuming that conflict resolution is yet another trainable EF in addition to updating and task-switching lexical ambiguity resolution abilities may also be enhanced, hypothetically, through conflict-resolution training tasks designed to target EFs central to overriding dominant biases and implementing cognitive control (provided the effects are large enough to observe improvement; this may be particularly true in clinical patients). Future research might test whether EF training, with the right tasks, could garner improvements in integration among topdown contextual and lexical sources of evidence, particularly when these latter sources give rise to multiple conflicting meanings. There are obvious implications for clinical patients with wordcomprehension deficits stemming from poor conflict-resolution abilities.

\section{REFERENCE RESOLUTION \\ Theory}

When conversational participants interact, they establish what is known as "common ground," or shared beliefs. Brown-Schmidt (2009) has demonstrated that variations in cognitive-control abilities can explain healthy individuals' occasional inattentiveness to common-ground information; that is, objects visually accessible only to the listener are occasionally (incorrectly) favored as a referential interpretation over objects accessible to both partners. Specifically, individual differences in conflict resolution may determine if a listener can successfully override perspectiveinappropriate interpretations of referential ambiguities uttered by their partner. As such, conflict resolution may predict how easily semantic and pragmatic information is integrated in order to rule out incorrect interpretations during natural dialogue.

Indeed, a study testing young children corroborates this account by showing that although 5 -year-olds can distinguish common versus privileged knowledge during conversation, the preference for their own perspectives - assessed by gaze duration to inappropriate privileged-ground alternatives - is predicted by measures of conflict resolution and inhibitory control (Stroop, a tapping task, and the bear/dragon puppet task), all of which require resolving among conflicting representations by overriding a dominant rule/bias (Nilsen and Graham, 2009). That is, children with poorer cognitive-control demonstrated exaggerated looking times to high-conflict referential alternatives inaccessible to the speaker but hidden (or "privileged") so that only the listener (the child) can see them (e.g., a small duck when "Look at the duck" is uttered and competes with the target that is common knowledge, i.e., a large duck). Namely, children with better performance on high-conflict conditions of an inhibitory control task were more likely to override their egocentric view and modify their behavior to be consistent with information shared by both communicative parties, and did so selectively for high-conflict items evidenced by spending less time gazing at inappropriate privileged-ground alternatives.

Adults occasionally show similar consideration of perspectiveinappropriate interpretations when a speaker utters a referential ambiguity, failing to be sensitive to common-ground information immediately. This behavior is also related to individual 
variation in conflict-resolution abilities. For instance, during one "visual-world" task (Brown-Schmidt, 2009), participants assisted the experimenter in revealing the identity of subject-privileged pictures on a display by answering the experimenter's questions. Generally, addressees consulted common-ground information to resolve temporarily ambiguous requests, like, What's above the horse with the glasses?, when two horses might be referenced, one wearing glasses and another wearing shoes. If the item above one of the horses (the horse with shoes) was previously grounded, then subjects directed their gaze toward the unmentioned target and the horse (with glasses) located below it, as the ambiguity unfolded. Crucially, however, the degree to which an addressee was able to use perspective information to avoid considering inappropriate interpretations (i.e., understanding the question to mean the already-revealed object) was determined by his Stroop performance. That is, subjects with better conflict-control were quicker to resolve referential conflict by directing their attention away from grounded items and toward previously unmentioned items.

Although conflict-resolution measures account for the individual differences in perspective-taking ability in children and adults, common-ground assessment likely requires multiple different kinds of EF (e.g., memory for perspective). However, it is important to note that the only experimental conditions predicted by Stroop performance are those that impose high conflict-resolution demands.

\section{Hypothesis}

This raises the question: if relevant EF skills can be targeted and enhanced via conflict-resolution training (for instance, using a training-appropriate version of the Stroop task as in BrownSchmidt, 2009), would individuals (particularly children) subsequently be less likely to consider unintended interpretations in cases of referential ambiguity? That is, one might hypothesize that EF training, within a process-specific conflict-resolution framework, will result in a generally sharper ability to promote relevant sources of information like context and pragmatics, and suppress currently irrelevant ones (e.g., one's privileged perspective) through top-down control.

Indeed, there is indirect yet tantalizing support for this. Work by Kloo and Perner (2003) provides evidence for far-transfer across structurally dissimilar tasks of information re-characterization within a theory of mind context in young children, who were either assigned to card-sorting training or false-belief (perspective taking) training. The card-sorting task involved categorizing cards with two distinct features (e.g., two yellow apples, one green apple), with the relevant dimension changing (from number to color) after each set of cards was fully sorted. The false-belief task required children to answer questions about a conflicting situation in which one puppet performed an action on another, but claimed that it, instead, acted on a different puppet. To assess the training-mediated effects of card-sorting and theory of mind, two novel assessments were implemented: the card-sorting transfer task included incorporating multiple rules for new cards (sort by number then color) and sorting an entirely different set of cards on novel dimensions. The false-belief-transfer measure was a traditional Sally-Ann task using the same puppets from training. Reciprocal far-transfer was observed for both types of training - individuals receiving false-belief training improved on card-sorting, and those trained on card-sorting showed benefits on the Sally-Ann task - suggesting the presence of a shared object redescription process. Note that a similar card-sorting task resulted in transfer to "task-switching" measures in a report of near-transfer highlighted earlier (Karbach and Kray, 2009). Both sets of results point to the malleability of EFs important for perspective taking, namely, object re-description (given by the Kloo and Perner findings) and task-switching (consistent with Karbach and Kray's work). To this end, task-switching ability is apt to overlap with conflict resolution (object re-description), as switching between multiple rules involves overriding old features and rules in favor of newly relevant ones, a type of information re-characterization that is a hallmark of conflict resolution. A carefully designed training regimen - for example, by comparing task-switching training with conflict-resolution training - may illuminate the overlapping contributions of each EF for each false-belief and perspective-taking tasks similar to those outlined above.

\section{VERBAL FLUENCY \\ Theory}

During language production, the ease with which a lexical item is generated depends partly on the degree of competition from other candidate words. Competition demands are particularly high when multiple semantically related words are equally plausible contenders for selection (a classic case of underdetermined representational conflict; see above discussion). Items with high versus low name-agreement, for instance, present different levels of conflict during naming tasks, such that low name-agreement items associated with many alternative labels (e.g., couch/sofa/loveseat) elicit more competition, reflected by longer naming latencies, thus requiring the use of VLPFC-mediated conflict resolution to select among the competing alternatives (Kan and Thompson-Schill, 2004; Novick et al., 2009). High name-agreement items (e.g., images that invoke a single label, like apple), by contrast, have fewer alternative labels to choose from, rendering them easier to access and produce, and thus, less dependent on conflict-resolution processes. Furthermore, selection costs are compounded when cases of high-competition (low name-agreement) are crossed with increased retrieval demands (e.g., low association-strength between a cue and its most accessible response), such that items with multiple weak associates are most difficult to output (Snyder et al., 2010).

This high-vs. low-name-agreement asymmetry has been examined in non-fluent aphasic patients with VLPFC damage - the same patients mentioned above who exhibit generally poor conflict resolution and cognitive control on a variety of non-linguistic conflict-resolution tasks like Stroop and the recent-no task. This population demonstrates exaggerated effects of production difficulty for high-competition conditions that require the recruitment of conflict-resolution resources, such that they take significantly longer or even fail to produce these items altogether relative to low-competition items (Novick et al., 2009). Patients with this neuroanatomical profile have difficulty with other verbal fluency tasks, including completing sentences when the options are openended (and therefore ambiguous), vs. when the to-be-completed fragments provide a highly constrained context, yielding little 
competition from possible alternative continuations (Robinson et al., 1998, 2005). Similarly, healthy speakers take longer to produce the names of pictured objects when they are presented in semantically homogeneous (e.g., snake, cow, dog, ant) vs. mixed contexts (e.g., snake, bus, axe, chair) due to the increase in lexicalsemantic competition among semantically related competitors (Belke et al., 2005). In one study, non-fluent aphasics with circumscribed VLPFC damage generated more errors when naming objects in homogeneous contexts; a companion neuroimaging experiment further showed that even healthy adults with a greater VLPFC response to naming under homogeneous conditions are prone to more naming errors compared to individuals with less VLPFC activation (Schnur et al., 2009).

\section{Hypothesis}

Careful consideration of the literature suggests that language production under conditions of conflict appears to be modulated by general EF abilities, like those governing conflict resolution on Stroop-like tasks. Consequently, training tasks tapping these same underlying neural networks may, hypothetically, be drawn on as tools to boost word selection abilities under elevated conflictresolution demands. The idea is that better conflict-resolution skills acquired through training might generalize to an increased ability to resolve among semantically related lexical items that compete for selection, carrying important implications for clinical interventions in populations with deficits in verbal fluency that accompany a more general deficit in conflict resolution.

Furthermore, training may also have consequences for selecting among competing alternative names during states of elevated anxiety. Indeed, one study reveals that more anxious individuals (evaluated by a composite score of anxious apprehension) are impaired relative to less anxious subjects when they must generate an associated verb (in response to a given noun) under high retrieval demands, an effect mediated by VLPFC (Snyder et al., 2010). This suggests that EF resources are depleted in cases of anxiety (Gray et al., 2002), which can negatively affect word selection processes under elevated $\mathrm{EF}$ demands (e.g., high-competition items). Future research on conflict-resolution training, therefore, might also address whether the right interventions can be used to offset such effects of anxiety and other deleterious affective states in both production and comprehension (but see Beilock and Carr, 2005).

\section{SUMMARY, CAVEATS, AND FUTURE DIRECTIONS}

Overall, we reviewed a sample of language tasks that depend heavily on posterior regions of left VLPFC, which support conflictresolution abilities in a variety of populations. Among these measures there is great overlap in the EF processes involved to carry them out successfully, whether it means employing conflictresolution to produce the right word, resolve lexical ambiguities, take a speaker's perspective to avoid errors in interpretation despite referential ambiguity, or recover from temporary misanalysis during sentence parsing. We believe that in view of these convergent findings, the theory that conflict resolution and cognitive-control contributes to language use may lead to the hypothesis that these domain-general conflict-control processes could be the target of extensive training regimens, the result of which could be attenuated processing difficulty during language use across a range of tasks, as indexed through measures of far-transfer. Such hypotheses are motivated also by the demonstration of positive transfer effects in non-linguistic cognitive domains following regimens targeting other EFs. This work could be particularly applicable to patients with lesions restricted to left posterior VLPFC, to determine (a) if their conflict-resolution performance changes on linguistic and non-linguistic tasks post-training, and (b) what new compensatory processes or brain systems they engage to support any observed performance increases (evaluated through pretest/posttest neuroimaging). There are similar implications for young children, whose comprehension might fail for similar reasons as the patients (i.e., deficits in cognitive control). Generally, this research program could suggest new inferences about the plasticity of the mind and brain, with respect to language processing especially, and the causal effects of language and cognition interactions.

Given prior evidence for far-transfer from WM training tasks to other measures such as task-switching, updating, and general fluid intelligence, the major goal that we are outlining, based on our theory of the role of left VLPFC and cognitive control in language processing, would be to design training studies in search of generalized effects to language measures, in hopes of mitigating difficulties under certain production and comprehension conditions during everyday language use. Except for a study conducted by our group on the effects of conflict-resolution training on syntactic ambiguity resolution, we are unaware of other research investigating whether broader improvements might be observed in language processing assessments in adults, both healthy and impaired, and even in young children. EF interventions might be particularly attractive in clinical arenas as a technique to remediate conflict-resolution deficits broadly construed, including how such impairments affect non-fluent production and comprehension difficulties under high-EF demands. Considering the patterns we reviewed suggesting a shared role for domain-general conflictresolution processes across a variety of language processing tasks, a common training regimen targeting this EF could, hypothetically, be successful in correcting problems observed in each of these tasks. Future research should test this, perhaps through various ways to evaluate transfer, including behavioral changes, changes in brain-activation patterns in regions commonly recruited across training and transfer tasks, changes in evoked response potentials (McLaughlin et al., 2004), changes in neural connectivity (Geva et al., 2011), changes in eye-movement patterns and reading-time latencies, or any combination of these measures.

\section{CAVEATS}

There are, however, important caveats to consider. Despite several instances of successful generalization to unpracticed tasks, some reports describe research efforts failing to observe transfer. One explanation for the absence of transfer findings may be that in at least one study, EF training was implemented casually, rather than consistently enough to actually tax trainees' EF abilities throughout the regimen (Owen et al., 2010). In this report, not all individuals in the training group received the same exposure to training, a "dosage-dependent" factor known to confer varying 
levels of transfer (Jaeggi et al., 2008). Another reason for failure to show transfer involves the use of performance-non-adaptive training tasks (regimens that maintain a constant level of difficulty, rather than keeping participants on the threshold of their best performance), despite strong evidence favoring such designs to facilitate transfer effects (Klingberg et al., 2005; Brehmer et al., 2011). Clearly more research is needed to determine what characterizes an appropriate training regimen, as well as how dependent transfer effects are on the amount of training an individual receives (Jaeggi et al., 2008, 2011). Finally, studies failing to show transfer might lack appropriate linking hypotheses between the types of EF required to perform certain tasks; these must be understood in order to design effective training regimens, which will ultimately inform how future intervention studies are implemented.

Furthermore, there appear to be important individual differences in training success (Chein and Morrison, 2010; Jaeggi et al., 2011), such that only certain individuals achieve performance increases on the training tasks over time, and thus demonstrate transfer to unpracticed measures shown through improved performance at retest (indeed, we observed this in our own training work). It is unclear if responders and non-responders can be categorized simply by baseline EF abilities, and these differences are unlikely due to motivational factors alone (Jaeggi et al., 2011; Novick et al., submitted for publication). So, future research should address who is most likely to benefit from training, how to identify these individuals, and how training protocols should be modified or tailored to maximize transfer across a range of groups and populations (see Shipstead et al., 2012).

Another remaining question concerns the lasting effects of training. Presumably, like physical fitness conditioning, the benefits of cognitive training do not persist indelibly without continued practice, though some have demonstrated maintained benefits three to six months after training ceased (Holmes et al., 2009; Jaeggi et al., 2011; Klingberg et al., 2005). Future work should address the long-term effects of cognitive conditioning, including the advantages of giving a periodic "booster training session" to reinstate the benefits after a regimen completion.

We included young children in our brief review of the role of conflict resolution in language use to illustrate a population whose poor EF abilities yield certain language-performance failures. However, research on training this population might proceed cautiously, particularly concerning language outcomes. The reason is that the protracted development of frontal cortex - although associated with suboptimal performance on cognitive-control (and relevant language) tasks - might actually confer certain advantages throughout development that overshadow the drawbacks. For instance, delayed PFC development - and by extension, delayed EF abilities - may bestow a benefit to certain aspects of cognitive development such as language acquisition (as opposed to language performance) and creativity (Thompson-Schill et al., 2009). There may be a complex tradeoff between bottom-up (data-driven) and top-down (rule-based) thinking in young children that may promote learning and social development. Therefore, if EF training is aimed at enhancing cognitive-control abilities, such interventions might have negative consequences, at least temporarily, for this population. Future work should address this concern, in addition to the long-term effects of training.
Finally, research examining healthy adults and patients with neurological disorders demonstrates that EF hinges on the involvement of a widespread network that comprises both cortical (e.g., $\mathrm{PFC}$, cingulate, and parietal) and subcortical (e.g., striatal) regions, clearly not just on prefrontal cortex alone (Corbetta and Shulman, 2002; Cools et al., 2007; Burgess et al., 2011; inter alia). This pattern is bolstered by training studies documenting the underlying neural signatures accompanying post-intervention differences, including increased activation of frontoparietal regions (Olesen et al., 2004); greater structural integrity evaluated by increased fiber tracts (white matter) connecting areas adjacent to intraparietal sulcus (Takeuchi et al., 2010); and an increase in the density of cortical dopamine receptors, perhaps linked to changes in striatal structures (McNab et al., 2009). Although behavioral and neuroimaging findings suggest domain-general processes in PFC that underlie cognitive-control functions across various conditions (Thompson-Schill et al., 2005), an intricate balance exists between PFC and subcortical regions that adjusts performance over different EFs (Cools et al., 2007). Such a cortical/subcortical tradeoff should be considered when choosing training and language-transfer tasks to maximize theoretical and functional-anatomical overlap, thereby increasing the prospect of transfer yield.

\section{FUTURE DIRECTIONS}

As we have highlighted, transfer might be expected only if the EFs (e.g., conflict resolution) underlying certain language tasks are targeted through training so as to affect shared processes that facilitate performance on particular language tasks (i.e., WM training tasks not involving conflict-resolution are not expected to confer transfer). Future work might continue to identify these functionalanatomical overlaps across different memory and language tasks. We believe however that there has been sufficient data accumulated to suggest a good candidate regimen targeting VLPFC-mediated conflict-resolution processes, which could affect certain language processing skills.

It is important to note that although we chose to focus on conflict-resolution functions given the extant data, this does not preclude the involvement of other EFs in the abovementioned language tasks (Miyake et al., 2000). To this end, the lack of mutual exclusivity of certain general cognitive processes should be considered when interpreting transfer effects within a process-specific framework, as multiple EFs might be confounded within a single training task; thus, changes in several EFs may be responsible for resultant improvements in outcome measures, a positive outcome if the goal is to show widespread transfer (e.g., Morrison and Chein, 2011; Shipstead et al., 2012). Likewise, in the examples cited above, the magnitude of the hypothesized transfer effects will likely be sensitive to the level of conflict-resolution required for each task. The amount of transfer will hinge on the degree to which underlying EFs are shared between training and assessment tasks, and this mechanistic overlap is probably influenced by both the relative involvement of a single trained EF and the extent to which other EFs are recruited in the training and outcome tasks. For example, re-characterization of representations on the high-conflict lure trials of the $n$-back task likely requires other EFs beyond just conflict resolution (e.g., monitoring, updating). 
Similarly, syntactic ambiguity resolution will, of course, rely on updating processes in addition to conflict resolution. Methodologically confounding EFs is an issue that plagues training studies, rendering it difficult to extricate distinct mechanisms entirely; however, by having linking hypotheses, EF overlap across tasks maximizes chances of successful transfer. Careful design of training regimens, including tasks performed by comparison groups for instance by maintaining minimal task differences between training and active-control tasks (e.g., a group completing the $n$-back task without the lure component) - can help elucidate the contribution of distinct EFs.

Correspondingly, the transfer conditions under which selective improvement is observed within an assessment task may mark those relying most on the trained EF. To maximize transfer, it is important to pinpoint the measures in the assessments that capture cognitive processes of interest. For instance, in our training experiment, we argued that the strongest indices of reinterpretation ability and real-time reanalysis respectively were accuracy to comprehension questions gaging lingering effects of misinterpretation and regression-path reading time in disambiguating sentence regions. Likewise, decreased gaze duration to privileged items in a common-ground assessment task, for example, probably involves information re-characterization, rendering this a candidate measure to observe conflict-resolution trainingrelated changes. The ability to make specific predictions for when and where transfer is selectively expected, as well as the conditions under which it is not, will ultimately lend important insight to the EFs affected during successful intervention when transfer effects are observed in studies carried out under proper linking assumptions and within a theoretically guided process-specific account.

Also worth mentioning is the contribution of several perhaps even overlapping - domain-general resources that may be recruited during language tasks not discussed here (e.g., mnemonic aspects of WM, maintenance, updating, taskswitching, etc). This should be carefully considered upon designing outcome language assessments that will be the target of transfer benefits. In fact, we strongly believe that verbal WM "span" processes, which involve maintenance, processing, and temporary storage components, must play a role in spoken language comprehension tasks in which the listener cannot review the input (as she can in normal reading) once it is spoken, without using mnemonic rehearsal strategies. This is likely true regardless of the presence of ambiguity or conflicting representations, and, indeed, verbal WM by itself has been shown to play a role in reading studies using a moving-window paradigm that does not permit rereading (Fedorenko et al., 2006). Thus, in future work it will be important to design training protocols using tasks that maximize a theoretical match between the cognitive (and neural) processes involved in assessment and training measures, including WM tasks that do not necessarily involve the conflict-resolution aspect of cognitive control, when appropriate.

Cognitive training may also provide a novel approach to understanding whether EFs are critical for a multitude of language uses. The degree to which training improvement predicts changes in language processing can reveal the EFs involved in each condition; if no transfer is observed in selective cases, one might conclude that the trained EFs do not significantly contribute to the processing of the particular language condition. This type of approach provides a powerful tool for choosing among several explanations for the same data set, where the best account of the data can be gleaned from the results of a well-designed training study that poses process-specific linking hypotheses. For example, some argue that the difficulty experienced while comprehending the meaning of abstract (compared to concrete) words hinges almost entirely on domain-general processes (Hoffman et al., 2010), while other accounts posit little to no contribution from EFs (Barsalou and Wiemer-Hastings, 2005; Rodríguez-Ferreiro et al., 2011). The opportunity exists, then, to investigate whether successful EF training permits better abstract-meaning selection.

Finally, it is important to consider a growing body of research demonstrating that balanced bilinguals enjoy certain cognitive advantages relative to their monolingual peers, as this work has important implications for language education and intervention. On tasks requiring cognitive control, some findings suggest that bilinguals outperform monolinguals selectively on trials inducing conflict across a range of tasks such as the Simon task (Bialystok et al., 2004). Other data patterns reveal a broader effect, namely that bilinguals are better at conflict monitoring: they perform faster on both conflict and non-conflict trials under high, but not low, conflict-monitoring conditions, in which subjects cannot predict when a conflict-related item type (an incongruent flanker trial) might occur because their appearance is equally probable relative to non-conflict trials (Costa et al., 2009). Regardless of the specifics, it has become increasingly clear that rich linguistic experience (akin to the rich cognitive experience achieved through training) benefits conflict-resolution and cognitive-control performance widely, perhaps due to bilinguals' consistent switching across the two language systems they know and/or their frequent suppression of one lexicon/grammar over another, thus placing a "premium" on EFs associated with updating, conflict resolution, and set-shifting (Martin-Rhee and Bialystok, 2008; Costa et al., 2009). In other words, lifelong bilingualism may be a naturalistic form of cognitive-control training. Indeed, future work should attempt to disentangle the various processing demands that are associated with being a bilingual speaker (e.g., frequent code switches) that might yield the putative cognitive-control advantage they show; such an understanding might help extract the various EFs, in addition to conflict resolution, that are at the heart of bilinguals' benefit. It will also be beneficial to know how bilinguals' cognitive-control advantage concerning conflict resolution or conflict monitoring influences this group's linguistic abilities on the conflict-related language tasks reviewed in this paper. For instance, does bilinguals' cognitive-control advantage result in a better ability to recover the correct interpretation of garden-path sentences, following a misanalysis? The answer to this question could suggest important inferences one could draw about the prospective impact that process-specific conflict-resolution training might have on this group.

Recent findings suggest that bilingualism confers protective benefits against cognitive decline: bilingual patients diagnosed with Alzheimer's disease (AD), who are matched on a range of factors (e.g., degree of cognitive impairment, symptomatic 
expression, demographic variables) to monolinguals with the same diagnosis, have significantly more brain atrophy in areas commonly examined to differentiate AD patients from healthy adults (Schweizer et al., 2011). The implication is that bilinguals may have greater "cognitive reserve" than would be predicted given the amount of neuropathology they exhibit; that is, the cognitive symptoms associated with $\mathrm{AD}$ may be delayed in this population because of their premorbid advantage. What about bilingual children and VLPFC patients? Are they "inoculated" from the cognitive-control deficits they are otherwise known for (in monolinguals) in terms of their non-linguistic and language processing abilities under high-conflict demands? If so, what behavioral mechanisms and neural systems do they recruit to compensate?

Furthermore, will cognitive-control training over the long-term yield similar protective benefits in monolinguals? Will their performance begin to approach that of (untrained) bilinguals? Will EF training confer comparable protection against normal age-related cognitive decline (Richmond et al., 2011), regardless of AD? These are open empirical questions and might be the focus of future longitudinal research. Also: to what extent does proficiency level matter in adults who have learned a second language, regarding the cognitive-control benefits they reap and the implications for intervention? Balanced bilinguals, as sketched above, enjoy certain advantages; presumably highly proficient (but unbalanced) bilinguals and those with lower proficiency levels will pattern somewhere in between the balanced group and the monolinguals regarding cognitive-control performance, depending on the relative processing demands associated with their proficiency levels. Where such bilinguals pattern can provide useful insight into the design of future training studies to bring these groups' performance ranges closer to approximate the balanced population. How much room is there for balanced bilinguals to gain from EF training? If a highly proficient group shows a similar cognitive-control advantage to that of bilinguals, then it may suggest the prospect of similar benefits (in terms of effect sizes) gained from training.

\section{REFERENCES}

Altmann, G. T., and Kamide, Y. (1999). Incremental interpretation at verbs: restricting the domain of subsequent reference. Cognition 73, 247-264.

Badre, D., and Wagner, A. D. (2007). Left ventrolateral prefrontal cortex and the cognitive control of memory. Neuropsychologia 45, 2883-2901.

Balota, D. A., and Faust, M. E. (2001). "Attention in dementia of the Alzheimer's type," in Handbook of Neuropsychology, eds F. Boller, and S. F. Cappa (New York: Elsevier Science), 51-80.

Barsalou, L. W., and Wiemer-Hastings, K. (2005). "Situating abstract concepts," in Grounding Cognition: The Role of Perception and Action in Memory, Language, and Thought, eds D. Pecher, and R. Zwaan (New York: Cambridge University Press), 129-163.
Bedny, M., Hulbert, J. C., and Thompson-Schill, S. L. (2007). Understanding words in context: the role of Broca's area in word comprehension. Brain Res. 1146, 101-114.

Beilock, S. L., and Carr, T. H. (2005). When high-powered people fail: working memory and "Choking Under Pressure" in math. Psychol. Sci. 16, 101-105.

Belke, E., Meyer, A., and Damian, M. F. (2005). Refractory effects in picture naming as assessed in a semantic blocking paradigm. Q. J. Exp. Psychol. 58, 667-692.

Bialystok, E., Craik, F. I. M., Klein, R., and Viswanathan, M. (2004). Bilingualism, aging, and cognitive control: evidence from the Simon task. Psychol. Aging 19, 290-303.

Bilenko, N. Y., Grindrod, C. M., Myers, E. B., and Blumstein, S. E.

Conversely, if a low-proficiency group that rarely switches between linguistic systems does not demonstrate a cognitive-control advantage compared to monolinguals, this would suggest opportunity for EF training to bestow benefits. If neither high- nor lowproficiency groups demonstrates a cognitive-control advantage, then perhaps learning a second language in adulthood does not enhance EF abilities similar to how early acquisition of two linguistic systems does. EF training could therefore be beneficial to unbalanced groups across a range of proficiency levels. Ultimately, future work in this area will clarify our understanding of the interplay between bilingualism, cognitive control, and the effects of training on language and other tasks that share cognitive processes.

\section{CLOSING REMARKS}

EF training holds promise to result in gains in cognition and language use in both production and comprehension domains, easing processing difficulty when multiple active and equally compelling representations are at odds (underdetermined representational conflict), or when dominant biases must be reined-in (prepotent conflict). Such interventions could potentially mitigate problems in language use under generally high conflict demands, not just in special populations (e.g., non-fluent aphasics with conflict-resolution deficits), but also in healthy individuals, including developing children, who experience occasional difficulty in reading, listening, or speaking due to heightened demands for cognitive control (in some cases perhaps due to resource depletion). Such research, provided reliable demonstrations of far-transfer, would add insight to our current understanding of how broad, non-linguistic cognitive abilities contribute to language use.

\section{ACKNOWLEDGMENTS}

This research was funded by the University of Maryland Center for Advanced Study of Language and an NSF IGERT Grant on Biological and Computational Foundations of Language Diversity (grant DGE-001465).

(2009). Neural correlates of semantic competition during processing of ambiguous words. J. Cogn. Neurosci. 21, 960-975.

Botvinick, M. M., Braver, T. S., Barch, D. M., Carter, C. S., and Cohen, J. D. (2001). Conflict monitoring and cognitive control. Psychol. Rev. 108 624-652.

Brehmer, Y., Rieckmann, A., Bellander, M., Westerberg, H., Fischer, H., and Bäckman, L. (2011). Neural correlates of training-related working-memory gains in old age. Neuroimage 58, 1110-1120.

Brown-Schmidt, S. (2009). The role of executive function in perspective taking during online language comprehension. Psychon. Bull. Rev. 16 893-900.

Burgess, G. C., Gray, J. R., Conway, A. R., and Braver, T. S. (2011). Neural mechanisms of interference control underlie the relationship between fluid intelligence and working memory span. J. Exp. Psychol. Gen. 140, 674-692.

Chein, J. M., and Morrison, A. B. (2010). Expanding the mind's workspace: training and transfer effects with a complex working memory span task. Psychon. Bull. Rev. 17, 193-199.

Christianson, K., Williams, C. C., Zacks, R. T., and Ferreira, F. (2006). Younger and older adults' "Goodenough" interpretations of gardenpath sentences. Discourse Process. 42, 205-238.

Cools, R., Sheridan, M., Jacobs, E. J., and D’Esposito, M. D. (2007). Impulsive personality predicts dopaminedependent changes in fronto-striatal activity during component processes of working memory. J. Neurosci. 27, 5506-5514. 
Copland, D. A., Sefe, G., Ashley, J., Hudson, C., and Chenery, H. J. (2009). Impaired semantic inhibition during lexical ambiguity repetition in Parkinson's disease. Cortex 45, 943-949.

Corbetta, M., and Shulman, G. L. (2002). Control of goal-directed and stimulus-driven attention in the brain. Nat. Rev. Neurosci. 3, 201-215.

Costa, A., Hernández, M., CostaFaidella, J., and Sebastián-Gallés, N. (2009). On the bilingual advantage in conflict processing: Now you see it, now you don't. Cognition 113, 135-149.

Dahlin, E., Neely, A. S., Larsson, A., Bäckman, L., and Nyberg, L. (2008). Transfer of learning after updating training mediated by the striatum. Science 320, 1510-1512.

D'Esposito, M., and Postle, B. R. (1999). The dependence of span and delayed-response performance on prefrontal cortex. Neuropsychologia 37, 1303-1315.

Fedorenko, E., Gibson, E., and Rohde, D. (2006). The nature of working memory capacity in sentence processing: evidence against domainspecific working memory resources. J. Mem. Lang. 54, 541-553.

Feuerstein, R. (1980). Cognitive modifiability in adolescence: cognitive structure and the effects of intervention. J. Spec. Educ. 15, 269-287.

Frazier, L., and Rayner, K. (1982). Making and correcting errors during sentence comprehension: eye movements in the analysis of structurally ambiguous sentences. Cogn. Psychol. 14, 178-210

Friedman, N. P., and Miyake, A. (2004). The relations among inhibition and interference cogntiive functions: a latent variable analysis. J. Exp. Psychol. Gen. 133, 101-135.

Geva, S., Correia, M., and Warburton, E. A. (2011). Diffusion tensor imaging in the study of language and aphasia. Aphasiology 25, 543-558.

Gray, J. R., Braver, T. S., and Raichle, M. E. (2002). Integration of emotion and cognition in the lateral prefrontal cortex. Proc. Natl. Acad. Sci. U.S.A. 99, 4115-4120.

Gray, J. R., Chabris, C. F., and Braver, T. S. (2003). Neural mechanisms of general fluid intelligence. $\mathrm{Nat}$. Neurosci. 6, 316-322.

Hamilton, A. C., and Martin, R. C. (2005). Dissociations among tasks involving inhibition: a single-case study. Cogn. Affect. Behav. Neurosci. 5, 1-13.

Hoffman, P., Jefferies, E., and Lambon Ralph, M. A. (2010). Ventrolateral prefrontal cortex plays an executive regulation role in comprehension of abstract words: convergent neuropsychological and rTMS evidence. J. Neurosci. 30, 15450-15456.

Holmes, J., Gathercole, S. E., and Dunning, D. L. (2009). Adaptive training leads to sustained enhancement of poor working memory in children. Dev. Sci. 12, F9-F15.

Hussey, E., Teubner-Rhodes, S., Dougherty, M., Bunting, M., and Novick, J. (2010). Improving garden-path recovery in healthy adults through cognitive control training. Talk Presented at the 16th Annual Conference on Architectures and Mechanisms for Language Processing, York, UK.

Huttenlocher, P. R., and Dabholkar, A. S. (1997). Regional differences in synaptogenesis in human cerebral cortex. J. Comp. Neurol. 387, 167-178.

Jaeggi, S. M., Buschkuehl, M., Jonides, J., and Perrig, W. J. (2008). Improving fluid intelligence with training on working memory. Proc. Natl. Acad. Sci. U.S.A. 105, 6829-6833.

Jaeggi, S. M., Buschkuehl, M., Jonides, J., and Shah, P. (2011). Short and long term benefits of cognitive training. Proc. Natl. Acad. Sci. U.S.A. 108 , 10081-10086.

January, D., Trueswell, J. C., and Thompson-Schill, S. L. (2009). Colocalization of Stroop and syntactic ambiguity resolution in Broca's Area: Implications for the neural basis of sentence processing. J. Cogn. Neurosci. 21, 2434-2444.

Jonides, J. (2004). How does practice make perfect? Nat. Neurosci. 7, 10-11.

Jonides, J., and Nee, D. E. (2006). Brain mechanisms of proactive interference in working memory. Neuroscience 139, 181-193.

Kan, I. P., and Thompson-Schill, S. L. (2004). Effect of name agreement on prefrontal activity during overt and covert picture naming. Cogn. Affect. Behav. Neurosci. 4, 43-57.

Kane, M. J., and Engle, R. W. (2000). Working memory capacity, proactive interference, and divided attention: Limits on long-term memory retrieval. J. Exp. Psychol. Learn. Mem. Cogn. 26, 333-358.

Karbach, J., and Kray, J. (2009). How useful is executive control training? Age differences in near and far transfer of task-switching training. Dev. Sci. 12, 978-990.

Khanna, M. M., and Boland, J. E. (2010). Children's use of language context in lexical ambiguity resolution. Q. J. Exp. Psychol. 63, 160-193.
Klingberg, T., Fernell, E., Olesen, P. J., Johnson, M., Gustafsson, P. Dahlström, K., Gillberg, C., Forssberg, H., and Westerberg, H. (2005). Computerized training of working memory in children with ADHD - a randomized, controlled trial. J. Am. Acad. Child Adolesc. Psychiatry 44 177-186.

Klingberg, T., Forssberg, H., and Westerberg, H. (2002). Increased brain activity in frontal and parietal cortex underlies the development of visuospatial working memory capacity during childhood. J. Cogn. Neurosci. 14, 1-10.

Kloo, D., and Perner, J. (2003). Training transfer between card sorting and false belief understanding: Helping children apply conflicting descriptions. Child Dev. 74, 1823-1839.

Li, S., Schmiedek, F., Huxhold, O., Röcke, C., Smith, J., and Lindenberger, U. (2008). Working memory plasticity in old age: Practice gain, transfer, and maintenance. Psychol. Aging 23, 731-742.

Martin-Rhee, M. M., and Bialystok, E. (2008). The development of two types of inhibitory control in monolingual and bilingual children. Biling. Lang. Cogn. 11, 81-93.

McLaughlin, J., Osterhout, L., and Kim, A. (2004). Neural correlates of second-language word learning: minimal instruction produces rapid change. Nat. Neurosci. 7, 703-704.

McNab, F., Varrone, A., Farde, L., Jucaite, A., Bystritsky, P., Forssberg, H., and Klingberg, T. (2009). Changes in cortical dopamine D1 receptor binding associated with cognitive training. Science 323, 800-803.

Milham, M. P., Banich, M. T., Webb, A., Barad, V., Cohen, N. J., Wszalek, T., and Kramer, A. F. (2001). The relative involvement of anterior cingulate and prefrontal cortex in attentional control depends on nature of conflict. Cogn. Brain Res. 12, 467-473.

Miller, E. K., and Cohen, J. D. (2001). An integrative theory of prefrontal cortex function. Annu. Rev. Neurosci. 24, 167-202.

Miyake, A., Friedman, N. P., Emerson, M. J., Witzki, A. H., Howerter, A., and Wager, T. D. (2000). The unity and diversity of executive functions and their contributions to complex "Frontal Lobe" tasks: a latent variable analysis. Cogn. Psychol. 41, 49-100.

Monsell, S. (1978). Recency, immediate recognition and reaction time. Cogn. Psychol. 10, 465-501.

Morrison, A. B., and Chein, J. M. (2011). Does working memory training work? The promise and challenges of enhancing cognition by training working memory. Psychon. Bull. Rev. $18,46-60$.

Nelson, J. K., Reuter-Lorenz, P. A., Sylvester, C. C., Jonides, J., and Smith, E. E. (2003). Dissociable neural mechanisms underlying response-based and familiaritybased conflict in working memory. Proc. Natl. Acad. Sci. U.S.A. 100, 11171-11175

Nilsen, E., and Graham, S. (2009). The relations between children's communicative perspective-taking and executive functioning. Cogn. Psychol. 58, 220-249.

Norman, W., and Shallice, T. (1986). "Attention to action: willed and automatic control of behavior," in Consciousness and Self Regulation: Advances in Research and Theory, Vol. 4, eds R. J. Davidson, R. Schwartz, and D. Shapiro (New York: Plenum), 1-18.

Novick, J. M., Kan, I. P., Trueswell, J. C., and Thompson-Schill, S. L. (2009). A case for conflict across multiple domains: Memory and language impairments follow damage to ventrolateral prefrontal cortex. Cogn. Neuropsychol. 26, 527-567.

Novick, J. M., Trueswell, J. C., and Thompson-Schill, S. L. (2005). Cognitive control and parsing: reexamining the role of Broca's area in sentence comprehension. Cogn. Affect. Behav. Neurosci. 5, 263-281.

Novick, J. M., Trueswell, J. C., and Thompson-Schill, S. L. (2010). Broca's area and language processing: evidence for the cognitive control connection. Lang. Linguist. Compass 4, 906-924.

Olesen, P., Westerberg, H., and Klingberg, T. (2004). Increased prefrontal and parietal brain activity after training of working memory. Nat. Neurosci. 7, 75-79.

Owen, A. M., Hampshire, A., Grahn, J. A., Stenton, R., Dajani, S., Burns, A. S., Howard, R. J., and Ballard, C. G. (2010). Putting brain training to the test. Nature 465, 775-778.

Persson, J., Welsh, K. M., Jonides, J., and Reuter-Lorenz, P. A. (2007). Cognitive fatigue of executive processes: interaction between interference resolution tasks. Neuropsychologia 45, 1571-1579.

Richmond, L., Morrison, A., Chein, J., and Olson, I. (2011). Working memory training and transfer in older adults. Psychol. Aging 26, 813-822.

Robinson, G., Blair, J., and Cipolotti, L. (1998). Dynamic aphasia: An 
inability to select between competing verbal responses? Brain 121, 77-89.

Robinson, G., Shallice, T., and Cipolotti, L. (2005). A failure of high level verbal response selection in progressive dynamic aphasia. Cogn. Neuropsychol. 22, 661-694.

Rodríguez-Ferreiro, J., Gennari, S. P., Davies, R., and Cuetos, F. (2011). Neural correlates of abstract verb processing. J. Cogn. Neurosci. 23, 106-118.

Schnur, T. T., Schwartz, M. F., Kimberg, D. Y., Hirshorn, E., Coslett, H. B., and Thompson-Schill, S. L. (2009). Localizing interference during naming: convergent neuroimaging and neuropsychological evidence for the function of Broca's area. Proc. Natl. Acad. Sci. U.S.A. 106, 322-327.

Schweizer, T. A., Ware, J., Fischer, C. E., Craik, F. I., and Bialystok, E. (2011). Bilingualism as a contributor to cognitive reserve: evidence from brain atrophy in Alzheimer's disease. Cortex 12, 8-15.

Shipstead, Z., Redick, T. S., and Engle, R. W. (2010). Does working memory training generalize? Psychol. Belg. 50, 245-276.

Shipstead, Z., Redick, T. S., and Engle, R. W. (2012). Is working memory training effective? Psychol. Bull. PMID: 22409508. [Epub ahead of print].

Smith, E. E., and Jonides, J. (1999). Storage and executive processes in the frontal lobes. Science 283, 1657-1661.

Snyder, H. R., Hutchison, N., Nyhus, E., Curran, T., Banich, M. T., O’Reilly, R. C., and Munakata, Y. (2010). Neural inhibition enables selection during language processing. Proc. Natl. Acad. Sci. U.S.A. 107, 16483-16488.

Staub, A., and Rayner, K. (2007). "Eye movements and on-line comprehension processes," in The Oxford Handbook of Psycholinguistics, ed. G. Gaskell (Oxford: Oxford University Press), 327-342.

Takeuchi, H., Sekiguchi, A., Taki, Y., Yokoyama, S., Yomogida, Y., Komuro, N., Yamanouchi, T., Suzuki, S., and Kawashima, R. (2010). Training of working memory impacts structural connectivity. J. Neurosci. 30, 3297-3303.

Tanenhaus, M. K. (2007). "Eye movements and spoken language processing," in Eye Movements: A Window on Mind and Brain, eds R. P. G. van Gompel, M. H. Fischer, W. S., Murray, and R. L. Hill (Oxford: Elsevier), 309-326.

Tanenhaus, M. K., Spivey-Knowlton, M. J., Eberhard, K. M., and Sedivy, J. E. (1995). Integration of visual and linguistic information in spoken language comprehension. Science 268, 1632-1634.

Thompson-Schill, S. L., Bedny, M., and Goldberg, R. F. (2005). The frontal lobes and the regulation of mental activity. Curr. Opin. Neurobiol. 15 219-224.

Thompson-Schill, S. L., Ramscar, M. and Chrysikou, E. G. (2009). Cognition without control: when a little frontal lobe goes a long way. Curr. Dir. Psychol. Sci. 18, 259-263.

Thompson-Schill, S. L., Swick, D., Farah, M. J., D’Esposito, M., Kan, I. P., and Knight, R. T. (1998). Verb generation in patients with focal frontal regions: a neuropsychological test of neuroimaging findings. Proc. Natl. Acad. Sci. U.S.A. 95, 15855-15860.

Trueswell, J. C., Sekerina, I., Hill, N. M., and Logrip, M. L. (1999). The kindergarten-path effect: studying on-line sentence processing in young children. Cognition 73, 89-134.

Van der Linden, D., Frese, M., and Meijman, T. F. (2003). Mental fatigue and the control of cognitive processes: effects on preservation and planning. Acta Psychol. (Amst) 113, 45-65.

Vuong, L. C., and Martin, R. C. (2011). LIFG-based attentional control and the resolution of lexical ambiguities in sentence context. Brain Lang. 116, 22-32.

Weighall, A. R. (2008). The kindergarten path effect revisited: children's use of context in processing structural ambiguities. J. Exp. Child. Psychol. 99, 75-95.

Westerberg, H., Jacobaeus, H., Hirvikoski, T., Clevberger, $\mathrm{P}$.
Ostensson, M. L., Bartfai, A., and Klingberg, T. (2007). Computerized working memory training after stroke - a pilot study. Brain Inj. 21, 21-29.

Ye, Z., and Zhou, X. (2009). Conflict control during sentence comprehension: fMRI evidence. Neuroimage 48, 280-290.

Conflict of Interest Statement: The authors declare that the research was conducted in the absence of any commercial or financial relationships that could be construed as a potential conflict of interest.

Received: 01 August 2011; accepted: 02 May 2012; published online: 21 May 2012.

Citation: Hussey EK and Novick JM (2012) The benefits of executive control training and the implications for language processing. Front. Psychology 3:158. doi: 10.3389/fpsyg.2012.00158

This article was submitted to Frontiers in Cognition, a specialty of Frontiers in Psychology.

Copyright (c) 2012 Hussey and Novick.

This is an open-access article distributed under the terms of the Creative Commons Attribution Non Commercial License, which permits non-commercial use, distribution, and reproduction in other forums, provided the original authors and source are credited. 